\title{
Scar Endometriosis: Case Report with Literature Review
}

\author{
Gupta P, Gupta S \\ Department of Obstetrics and Gynaecology, Post Graduate Institute of Medical Sciences and Research, ESIC, \\ Basaidarapur, New Delhi, India.
}

Received: December 15, 2013; Accepted: July 2, 2014

\begin{abstract}
Endometriosis is defined as presence of functioning endometrial tissue outside the uterine cavity. Endometriosis can sometimes occur in a previous surgical scar. Scar endometriosis is rare and difficult to diagnose. It mostly follows obstetrical and gynecological surgeries. This condition is often confused with other surgical conditions. We are reporting one case of scar endometriosis involving rectus sheath following cesarean section. The patient required wide surgical excision of the lesion. The pathogenesis, diagnosis and treatment of this rare condition are being discussed.
\end{abstract}

Keywords: abdominal wall; endometrioma; endometriosis; scar endometriosis.

\section{INTRODUCTION}

Endometriosis is defined as the presence or growth of ectopic endometrial tissue. ${ }^{1}$ Affecting an estimated 89 million women of reproductive age worldwide, endometriosis occurs in $5 \%$ to $10 \%$ of all women, often resulting in debilitating pain and infertility. However, extra pelvic endometriosis is an uncommon disorder and difficult to diagnose. The various sites for extra pelvic endometriosis are bladder, kidney, bowel, omentum, lymph nodes, lungs, pleura, extremities, umbilicus, hernia sacs, and abdominal wall. ${ }^{2}$ Abdominal wall endometrioma often develop in previous surgical scars but there is a case report of a spontaneous occurrence also. ${ }^{3}$ Majority of the scar endometriosis have been reported after obstetrical or gynecological procedures such as cesarean delivery, hysterotomy, hysterectomy, episiotomy, and tubal ligations but few case reports are following appendicectomy, in the laparoscopic trocar tract, amniocentesis needle tract. ${ }^{4-6}$ Scar endometriosis patients are often referred to the general surgeons because the clinical presentation suggests a surgical cause. In a study by Blanco et $\mathrm{al}^{7}$ the diagnosis was initially confused with inguinal hernia, incisional

\section{CORRESPONDENCE}

Dr Pratiksha Gupta

Department of Obstetrics and Gynaecology,

Post Graduate Institute of Medical Sciences and Research, ESIC,

Basaidarapur, New Delhi, India.

Email: drpratiksha@gmail.com

Phone: $+91-9871128703$ hernia and abdominal wall tumor. This entity can result in unnecessary procedures, delayed or misdiagnosis, and can cause emotional and physical distress to the patient. The present study describes a case of scar endometriosis, and reviews the literature to elucidate physical signs and symptoms that may lead to earlier diagnosis and prompt treatment.

\section{CASE}

Twenty eight year old female patient reported to our gynecology out patient in the month of May 2011, with chief complaints of swelling and pain in previous caesarian scar. Obstetrical history - she was P2L2, with one previous lower segment caesarian section done three years ago, for fetal distress. Postoperative period was uneventful. Since two years, she was having mass of $3 \times 3 \mathrm{~cm}$, at the region of left side of caesarian scar, with dull aching constant pain at the site of swelling without any radiation and association with menstrual cycle. This pain used to get relieved for sometime on taking some analgesics. She reported to our institute three months back.

General examination was within normal limits. Per abdominal examination revealed a brownish bluish mass of $3 \times 3 \mathrm{~cm}$, at left extreme side of Pfannensteil caesarian scar with slight tenderness, firm consistency, with restricted mobility (Figure 1). 


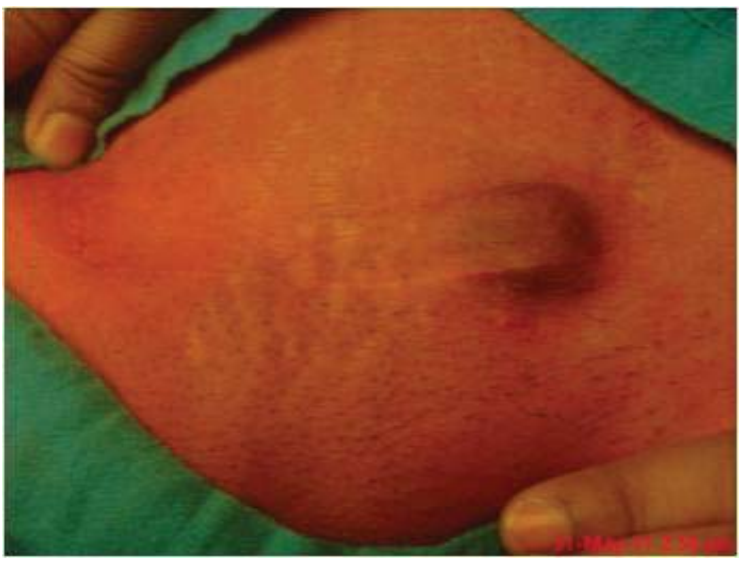

Figure 1. Bluish brownish swelling.

A probable diagnosis of scar endometriosis was made, and was planned for surgical excision. All hematological investigations were within normal limits. Wide excision of the endometriotic tissue was done. Stitches were removed on postoperative day 8. Stitch line has healed without any recurrence. Histopathological findings confirmed the diagnosis of scar endometriosis.

Hematoxylin and Eosin photomicrograph showing tissue surrounding benign endometrial glands and stroma consistent with endometriosis (Figure 2).

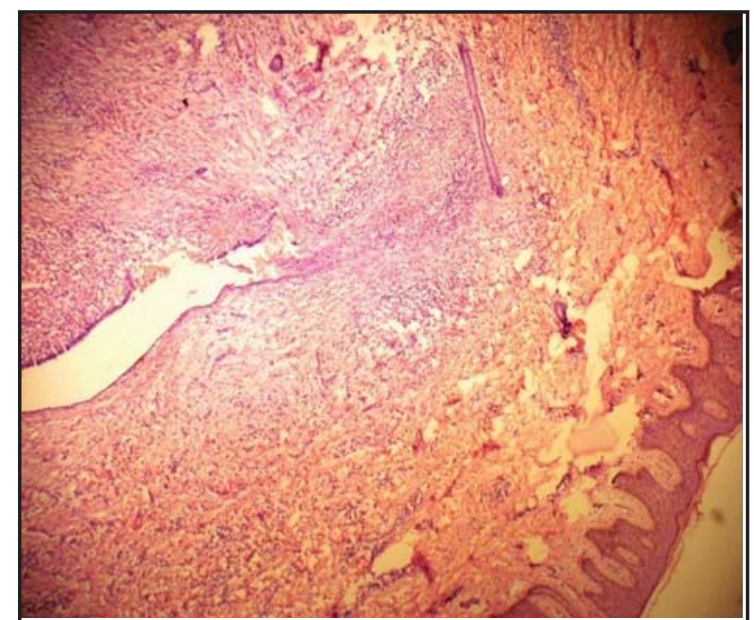

Figure 2. Histopathology of resected specimen showing endometrial glands.

It shows endometrial tissue with haemorrhage in upper right corner and a dilated endometrial gland in the lower right corner, showing endometrial gland, epidermis. Endometriosis is defined by occurrence of endometrial-like epithelium and stroma outside the uterine cavity. This condition is commonly seen in females of reproductive age. Grossly, endometriosis may present as small, dark red, black or bluish cysts or nodules on the surface of peritoneal and pelvic organs. Histologically, endometriosis is characterized by the ectopic presence of endometrial-like glands, spindled endometrial stroma and Hemosiderin deposition either within the macrophages or in the stroma (Figure 2). In many cases, this diagnostic triad is not present, or hemorrhage, foamy cells and Hemosiderin-laden macrophages may obscure the glands and stroma. When this occurs, the diagnosis may be suggested but histological confirmation may not be possible.

\section{COMMENT}

Endometrioma is a well-circumscribed mass of endometriosis. Abdominal wall endometrioma presents as a painful swelling resembling surgical lesions such as hernias, hematomas, granulomas, abscess and tumors. Therefore, that is why these cases generally first report to general surgeons. Scar endometriosis most commonly occurs after operation on the uterus and tubes. Incidence of scar endometriosis following Hysterotomy is 1.08$2 \%$ whereas after cesarean section the incidence is $0.03-0.4 \%{ }^{6}$ The reason for higher incidence after Hysterotomy has been given as the early Decidua has more pleuripotential capabilities and can result in cellular replication producing endometrioma. Time interval between operation and presentation has varied from 3 months to 10 years in different series. The etiology of abdominal wall endometrioma is thought to be a result of transportation of endometrial tissue during surgical procedures and subsequently stimulated by estrogen to produce endometrioma. The simultaneous occurrence of pelvic endometriosis with scar endometriosis is infrequent. ${ }^{6}$ Our patient also did not have associated pelvic endometriosis. Preoperative diagnosis is difficult to make and sometimes the diagnosis is made after excision only. We could make provisional preoperative diagnosis due to the discoloration and clinical experience. Various diagnostic methods have been described in the literature. Until recently the use of Ultrasonography (USG) has hardly been reported in detail and anecdotal reports have described it as nonspecific, can give a varied picture of hypo echoic mass with scattered internal echoes. Recently a large 
series of 12 patients where USG and color Doppler substantially contributed to the correct preoperative diagnosis $^{8}$ and authors suggest that sonographic and color Doppler when combined with clinical data may substantially contribute to the preoperative diagnosis. FNAC has been reported to be accurate in diagnosis but in a recent report by Dwivedi et al. FNAC was not diagnostic in any of the four patients who underwent this procedure. ${ }^{9,10}$ Anecdotal studies have mentioned the use of computed tomography (CT) and magnetic resonance imaging (MRI) in making a diagnosis. CT usually shows a solid, well-circumscribed mass. MRI can be more helpful when the lesion is small because of its high spatial resolution, furthermore it perform better than CT scan in detecting the planes between muscles and abdominal subcutaneous tissue. ${ }^{11}$ Treatment of choice is wide excision of the lesion and may sometimes require mesh placement as was done in our two patients. Medical treatment with the use of Progestogens, oral contraceptive pills, and Danazol is not effective and gives only partial relief in symptoms. Recently there has been report of use of Gonadotrophin agonist but only with the prompt improvement in symptoms with no change in the lesion size. ${ }^{12}$ These patients need to be followed up because of the chances of recurrence, which require re-excision. In cases of continual recurrence, possibility of malignancy should be kept in mind. To prevent the occurrence of scar endometriosis it has been suggested that at the end of surgery especially on uterus and tubes, the abdominal wall wound should be cleaned thoroughly and irrigated vigorously with high jet solution before closure. ${ }^{13}$

\section{CONCLUSIONS}

One should have high index of suspicion of scar endometriosis when a woman presents with a painful swelling in the abdominal scar especially with a history of previous gynecological or obstetrical surgery. This condition can be confused with other surgical conditions. Efforts should be made to make a preoperative diagnosis with the help of imaging techniques and FNAC. Wide excision is the treatment of choice.

\section{DISCLOSURE}

The authors report no conflicts of interest in this work.

No violation of human rights and safety.

Funding: Nil

\section{REFERENCES}

1. Goel P, Sood SS, Romilla, Dalal A. Cesarean scar endometriosis: report of two cases. Indian J Med Sci. 2005;59:495-8.

2. Wolf G, Singh K. Cesarean scar endometriosis: a review. Obstet Gynecol Surv. 1989; 44:89-95.

3. Ideyi SC, Schein M, Niazi M, Gerst PH. Spontaneous endometriosis of the abdominal wall. Dig Surg. 2003;20:246-

4. Padmanabhan LD, Mhaskar R, Mhaskar A. Scar endometriosis. J Obstet Gynaecol India. 2003;53:59-61.

5. Bhowmick RN, Paul P, Dutta S, Roy B. Endometriosis of laparotomy scar. J Obstet Gynaecol India. 1986;36:130-2.
6. Chatterjee SK Scar endometriosis: a clinicopathological study of 17 cases. Obstet Gynecol. 1980;56:81-4.

7. Blanco RG, Parithivel VS, Shah AK, Gumbs MA, Schein M, Gerst PH. Abdominal wall endometriomas. Am J Surg. 2003;185:596-8

8. Francica G, Giardiello C, Angelone G, Cristaino S, Finelli R, Tramontano G. Abdominal wall endometriomas near cesarean delivery scars: sonographic and color doppler findings in a series of 12 patients. J Ultrasound Med. 2000;22:1041-7.

9. Simsir A, Thorner K, Waisman J, Cangiarella J. Endometriosis in abdominal scars: a report of three cases diagnosed by fine needle aspiration biopsy. Am Surg. 2001;67:984-6.

10. Dwivedi AJ, Agarwal SN, Silva YJ. Abdominal wall endometriomas. Dig Dis Sci. 2002;47:456-61. 